\title{
Agrandamiento gingival farmacoinducido: Serie de casos
}

\author{
Drug-induced gingival enlargement: Series of cases \\ Isabella Manzur-Villalobos ${ }^{*}$ orcid.org/0000-0003-1979-7454 \\ Iván Alejandro Díaz-Rengifo² orcid.org/0000-0003-3959-8196 \\ Daniela Manzur-Villalobos ${ }^{1}$ orcid.org/0000-0002-5752-4070 \\ Antonio José Díaz-Caballero' ${ }^{1}$ orcid.org/0000-0001-9693-2969
}

1 Universidad de Cartagena. Cartagena, Colombia.

2 Colegio Mayor Nuestra Señora del Rosario. Bogotá, Colombia.

Fecha de recepción: Abril 8- $2017 \quad$ Fecha de revisión: Noviembre 22 - $2017 \quad$ Fecha de aceptación: Diciembre 21 - 2017

Manzur-Villalobos I, Díaz-Rengifo IA, Manzur-Villalobos D, Díaz-Caballero AJ. Agrandamiento gingival farmacoinducido: Serie de casos. Univ. Salud. 2018;20(1):89-96. DOI: http://dx.doi.org/10.22267/rus.182001.113

\section{Resumen}

Introducción: El agrandamiento gingival (AG) es una condición benigna de la cavidad oral que se caracteriza por el crecimiento excesivo en masa y volumen de la gingiva. Esta lesión no es únicamente provocada por factores hereditarios ni de deficiente higiene oral, sino también por ingesta de medicamentos, entre los cuales se encuentran fármacos antihipertensivos, anticonvulsivantes e inmunosupresores. Objetivo: Sensibilizar respecto a la prevención o atención temprana en pacientes con patologías que ameritan el uso de antihipertensivos y anticonvulsivantes, conjuntamente con el odontólogo para tratar o evitar el agrandamiento gingival fármaco inducido (AGFI) Materiales y métodos: Se reporta una serie de casos clínicos de pacientes con agrandamiento gingival por distintos fármacos, entre los que se encuentran fenitoina, amlodipino y nifedipino; se aplicaron medidas de fase higiénica periodontal y gingivectomía para obtener mejores efectos. Resultados: Se obtuvieron resultados satisfactorios con una disminución considerable del AGFI. Conclusiones: Es importante el manejo integral en conjunto con el médico tratante para realizar seguimiento del fármaco que pueda encontrarse generando agrandamiento gingival. Emplear un abordaje inicial con estrategias de higiene periodontal, y en casos severos y como último recurso la intervención quirúrgica periodontal con gingivectomía y gingivoplastia.

Palabras clave: Sobrecrecimiento gingival; antihipertensivos; inmunosupresores; anticonvulsivantes; gingivectomía. (Fuente: DeCS, Bireme).

\begin{abstract}
Introduction: Gingival enlargement (GA) is a benign condition of the oral cavity that is characterized by the excessive growth of the gingiva in mass and volume. This lesion is not only caused by hereditary factors or poor oral hygiene, but also by the intake of medications, including antihypertensive, anticonvulsant and immunosuppressive drugs. Objective: To sensitize the prevention or early care in patients with pathologies that merit the use of antihypertensive and anticonvulsants in conjunction with the dentist, to treat or avoid the drug-induced gingival enlargement (DIGE). Materials and methods: A series of clinical cases of patients with gingival enlargement by various drugs are reported, including Phenytoin, Amlodipine and Nifedipine. Periodontal and gingivectomy hygienic phase measures were applied to obtain better effects. Results: Satisfactory results were obtained with a considerable decrease in DIGE. Conclusions: The integral management is important in conjunction with the treating physician to follow up the drug that can be generating gingival enlargement. It is necessary to employ an initial approach with strategies of periodontal hygiene, and in severe cases and, as last resort, the periodontal surgery with gingivectomy and gingivoplasty.
\end{abstract}


Keywords: Gingival overgrowth; antihypertensive agents; inmunosupressive agents; anticonvulsants, gingivectomy. (Source: DeCS, Bireme).

\section{Introducción}

Se entiende por agrandamiento gingival (AG) el incremento en masa y volumen del tejido gingival, que puede variar en severidad(1). Se considera una condición benigna de la cavidad oral, por lo general de manejo rutinario, que logra regularse con medidas simples de control de biopelícula(2). El AG puede ser producido por diversas condiciones clínicas, hereditarias o deficiente higiene oral; esta última es la que está más asociada a su desarrollo debido a los cambios inflamatorios causados por el acúmulo de placa bacteriana ${ }^{(1,3)}$; sin embargo, también puede estar asociado al uso prolongado de algunos fármacos utilizados para tratar enfermedades crónicas, por esto, el objeto de este análisis de casos es el agrandamiento gingival inducido por fármacos (AGFI).

El AG inicia a manera de un agrandamiento nodular firme en la papila interdental(1), el cual puede extenderse coronalmente y generar problemas durante la masticación o el habla. El aumento progresivo del tejido gingival facilita la formación de bolsas periodontales produciendo un ambiente favorable para la colonización bacteriana que es difícil de retirar por medio del cepillado habitual(1,2). Al presentarse en el sector anterior condiciona igualmente, problemas estéticos y de salud preocupantes para el paciente(1). Histológicamente se encuentra un aumento excesivo de matriz extracelular, especialmente colágeno y glicosaminoglicanos, acompañado de un incremento en el número de fibroblastos y presencia de acantosis de la capa epitelial de la encía( ${ }^{(2,3)}$.

Existen diversos medicamentos que pueden condicionar AGFI como reacción adversa, sin embargo se destacan algunos grupos farmacológicos como los antihipertensivos, anticonvulsivantes e inmunosupresores $(3,4)$, los cuales pueden encontrarse en asociación para el tratamiento de patologías como Lupus Eritematoso Sistémico, dificultando en ciertos casos la identificación del medicamento que está relacionado directamente con el AG. Este efecto aparece usualmente después del uso prologando del medicamento o debido al consumo de altas. Las distintas revisiones de la literatura sugieren que el AGFI aparece posterior a 1-3 meses de consumo del medicamento(2), manifestándose como un abultamiento local o generalizado del tejido gingival con mayor tendencia a presentarse en las caras anteriores y en las superficies vestibulares ${ }^{(5)}$.

Dentro de los grupos farmacológicos más asociados al AGFI se encuentran los antihipertensivos de tipo bloqueadores de

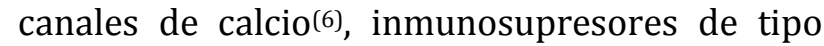
ciclosporina o azatioprina(7) y entre los anticonvulsivantes principalmente la fenitoína( ${ }^{(8)}$. Una vez identificado el agente asociado al AGFI debe evaluarse la posibilidad de disminución de la dosis empleada o el reemplazo de la medicación de acuerdo a las necesidades y beneficios del paciente; sin embargo, al tratarse de medicamentos para enfermedades de carácter crónico, ésta estrategia se encuentra limitada y puede ocurrir que incluso después de disminuir su dosis se mantenga el agrandamiento gingival y se requiera complementar con medidas de higiene oral adecuadas, iniciar con una fase higiénica exhaustiva para lograr una disminución de la colonización bacteriana y del edema tisular previo a la realización de intervenciones quirúrgicas como gingivectomía del sitio comprometido(8).

La epilepsia, afecta al 1\% de la población mundial y requiere del uso de fármacos antiepilépticos o anticonvulsivantes para lograr su control, dentro de estos, la fenitoína actúa como un bloqueador selectivo de los canales de sodio sensibles al voltaje y constituye uno de los fármacos más empleados por su capacidad en el control de crisis focales y generalizadas, a pesar de sus amplias interacciones y variabilidad interindividual(9). Los calcioantagonistas y la fenitoína se han relacionado con el AGFI como uno de sus efectos adversos, los cuales se incluyen dentro de las enfermedades farmacoinducidas en la cavidad 
oral como aquellas que afectan a la encía con hallazgos de hiperplasia; el mecanismo por el cual se produce dicho efecto no es completamente claro $^{(10)}$.

La hipertensión arterial es una condición frecuente en la edad adulta y los calcioantagonistas constituyen una de las principales alternativas farmacológicas de manejo. Medicamentos como el amlodipino son formulados en $65 \%$ de los pacientes en países como Cuba(11). Los bloqueadores de canales de calcio o calcioantagonistas corresponden a una familia farmacológica inicialmente desarrollada en los años 60's con efecto hipotensor y acciones a nivel cardiovascular que los han posicionado como fármacos de primera línea en el tratamiento de la hipertensión arterial; corresponden a un grupo heterogéneo de fármacos clasificados de acuerdo a la estructura química dihidropiridínica en no dihidropiridinas: verapamilo y diltiazem; y dihidropiridinas: nifedipino, amlodipino, nicardipino, entre otros. Su mecanismo de acción se relaciona con la inhibición de canales de calcio dependiente de voltaje tipo $\mathrm{L}$, a nivel del músculo liso del endotelio vascular y cardíaco, generando una reducción en el influjo intracelular de calcio por ende, favoreciendo la vasodilatación a nivel arteriolar de forma más selectiva para los bloqueadores de calcio dihidropiridínicos(12).

En el presente reporte se describe una serie de casos clínicos de AGFI, los cuales incluso después de haber realizado todas las medidas de interconsulta médica, adecuada higiene oral, motivación al paciente y fase higiénica, no se obtuvo disminución del aumento y abultamiento de la encía, por lo cual se realizó gingivectomía para garantizar una buena salud de los tejidos periodontales de los pacientes. El objetivo es aportar a la comprensión y sensibilización respecto a la prevención o atención temprana de agrandamiento gingival en pacientes con patologías que ameritan el uso de antihipertensivos y anticonvulsivantes.

\section{Consideraciones éticas}

Los pacientes participaron voluntariamente del estudio, fueron notificados de los alcances del mismo y firmaron consentimiento informado donde autorizaron la reproducción y publicación de fotografías. Adicional a esto, todos los pacientes que requirieron intervención quirúrgica firmaron el consentimiento informado donde se relacionaban todos los riesgos que podían ocurrir durante la intervención.

\section{Materiales y métodos}

Los siguientes casos que se presentarán a continuación corresponden a pacientes que acudieron a consulta odontológica con periodoncista, por presentar incomodidad en las encías. Con todos los pacientes se llevó a cabo el siguiente protocolo de intervención: primero se realizó historia de la enfermedad actual y antecedentes familiares y personales y examen clínico extraoral e intraoral.

Para el tratamiento en primera instancia se enseñó a los pacientes medidas de educación en higiene oral y técnicas de cepillado durante cuatro sesiones de fase higiénica periodontal. Sin embargo, no se evidenció disminución de la encía marginal, por lo cual se estableció el agrandamiento gingival como primer diagnóstico presuntivo.

Posterior a esto, el periodoncista procedió a realizar una gingivectomía en la zona del agrandamiento gingival para obtener una mejor estética y funcionalidad, para esto se inició la intervención con aplicación de anestesia local en el maxilar inferior, realizando una incisión a bisel externo tipo gingivectomía con un electrobisturí; el procedimiento produjo en los cuatro pacientes ligero sangrado gingival, sin complicaciones.

\section{Reporte de casos clínicos}

\section{Caso clínico 1}

Paciente de sexo masculino de 60 años de edad, acudió a la consulta por presentar incomodidad de las encías en el sector inferior (Figura 1). Refirió tener antecedentes de hipertensión arterial hace 15 años, en manejo crónico con amlodipino $10 \mathrm{mg}$ /día desde el momento de inicio de tratamiento. Al realizar examen clínico se observó aumento el volumen de la encía marginal y papilar, de aspecto lobulado, y de color rosa 
pálido, excepto en encía papilar distal de OD\#42. Asimismo, se observó hiperpigmentación melánica en encía adherida del maxilar inferior (Figura 1A). El procedimiento quirúrgico se realizó bajo anestesia local mediante incisión a bisel externo con electrobisturí, el paciente presentó ligero sangrado sin complicaciones (Figura 1B).

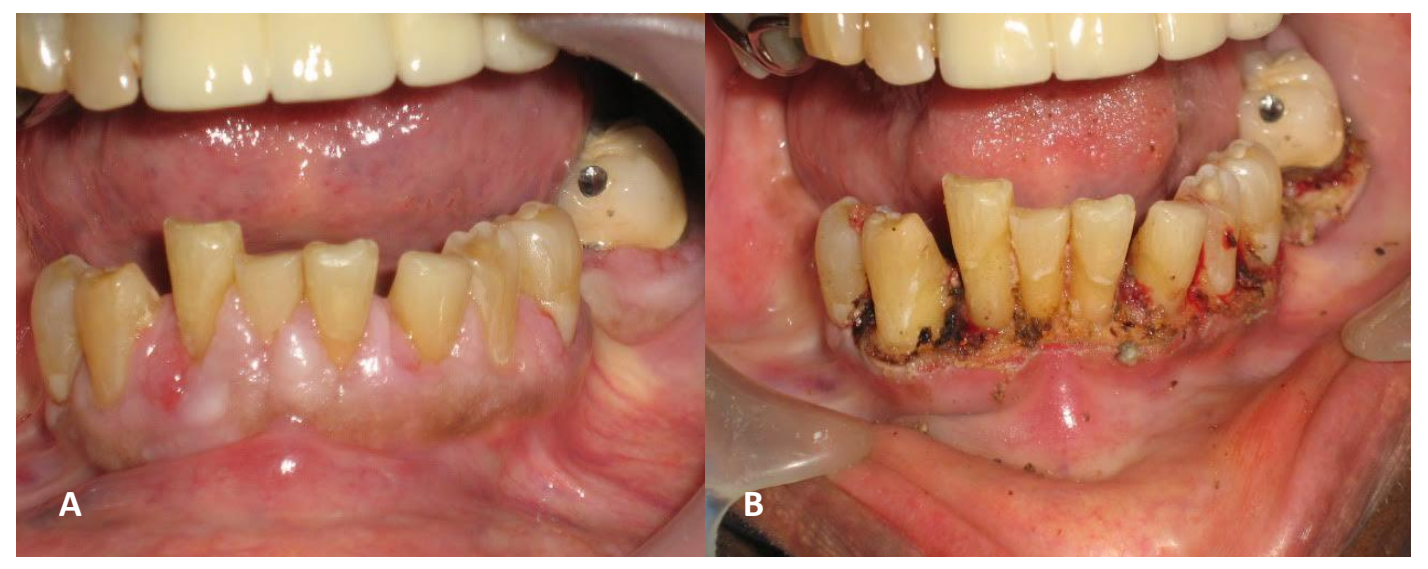

Figura 1. A) Aumento del volumen de la encía marginal y papilar, hiperpigmentación melánica en encía adherida del maxilar inferior. B) Gingivectomía realizada

\section{Caso clínico 2}

Paciente de sexo masculino de 65 años de edad quien refirió en la consulta odontológica sobrecrecimiento gingival en maxilar superior e inferior. Manifestó tener antecedentes de epilepsia hace aproximadamente 8 años, tratada con fenitoína (difenilhidantoína) 300mg/12 horas.

Al realizar examen clínico se observó aumento del volumen de la encía marginal y papilar y encía adherida tanto en maxilar superior como inferior, de aspecto lobulado, y de color rojo intenso. Ausencia de OD\# 11, 31; múltiples recesiones gingivales en OD\# 13, 12, 21, 22, 23, 32, 34, 35, 44, 45 de aproximadamente de 3 a $6 \mathrm{~mm}$. Además, se pudo evidenciar el acúmulo de placa bacteriana presente en las superficies interproximales de OD\# 13, 32, con un índice de placa bacteriana de $30 \%$ según el índice de O’lear(13) (Figura 2). El procedimiento quirúrgico se realizó sin complicaciones.

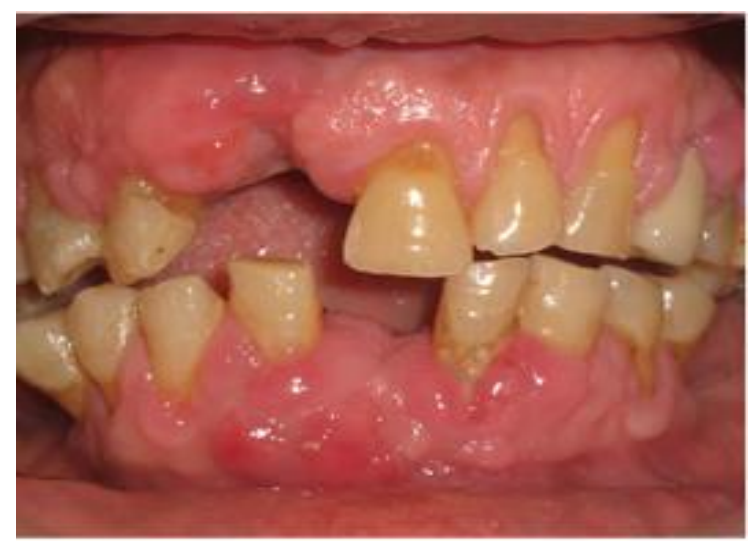

Figura 2. Paciente con aumento del volumen de la encía marginal y papilar y encía adherida en maxilares

\section{Caso clínico 3}

Paciente de sexo masculino de 67 años quien acudió a consulta odontológica con periodoncista debido a incomodidad por presencia de abultamiento en encías inferiores (Figura 3). Refirió tener antecedentes de hipertensión arterial hace más de 20 años, tratada con nifedipino $10 \mathrm{mg} / 8$ horas. Al realizar examen clínico se observó aumento del volumen de la encía marginal, papilar y encía adherida tanto en 
maxilar inferior, específicamente a nivel de OD\# $33,41,44,45$, de aspecto lobulado bastante marcado, y de color rojo intenso. Además, se encontró ausencia de OD\# 42; movilidad dentaria en OD\# 41. La encía marginal tenía aspecto lobulado, color rosa pálido en maxilar superior, a nivel de OD\# 12, 13, 21, 22 (Figura 3A). El procedimiento quirúrgico se realizó sin complicaciones. Luego de 15 días de evolución, se evidenció ausencia de volumen de encía y tejidos sanos (Figura 3B).

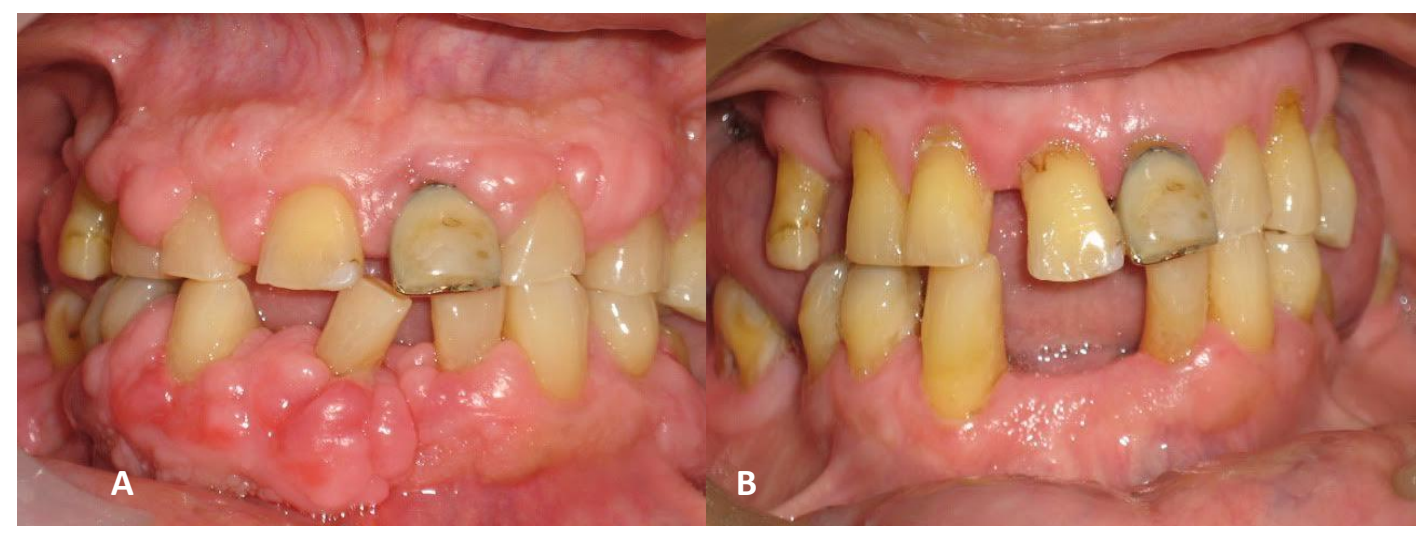

Figura 3. A) Paciente con aumento del volumen de la encía marginal, papilar y encía adherida en maxilares, ausencia de OD\# 42 y movilidad dentaria en OD\# 41. B) Registro fotográfico posterior a gingivectomía

\section{Caso clínico 4}

Paciente de sexo masculino de 59 años de edad, con agrandamiento gingival en maxilar superior, a nivel de los órganos dentarios pilares para una prótesis total (Figura 4). Refirió tener antecedente de hipertensión arterial hace 9 años en tratamiento con nifedipino $10 \mathrm{mg} / 8$ horas, aproximadamente hace 6 años. Al examen clínico se observó aumento del volumen de la encía marginal y papilar, de aspecto lobulado, y de color rosa pálido a nivel de OD\# 12,13, 22, 23, 24; excepto en encía papilar distal de OD\# 12, la cual se encuentra eritematosa y edematizada. Se observan OD\# 12 13, 22, 23, 24 previamente tallados y con presencia de núcleos colados (Figura 4A). El procedimiento quirúrgico se realizó sin complicaciones (Figura 4B).

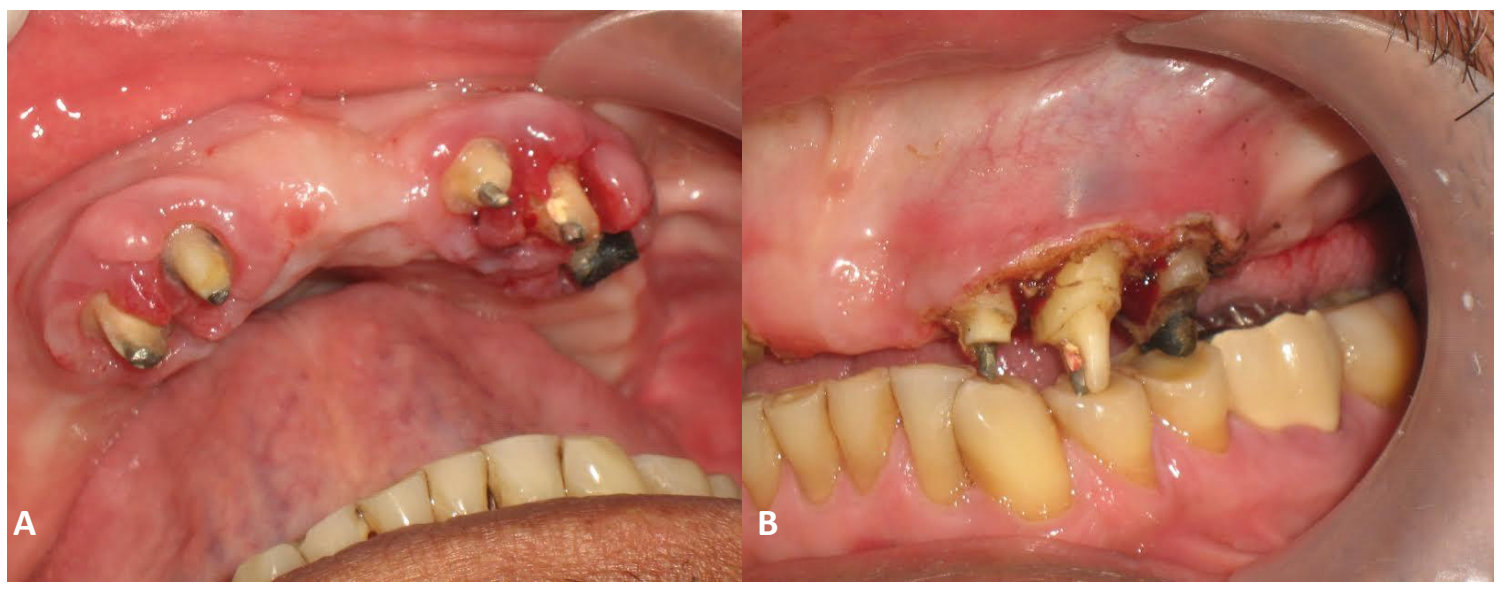

Figura 4. A) Paciente con aumento del volumen de la encía marginal y papilar. B) Gingivectomía realizada bajo anestesia local 


\section{Discusión}

Dentro del AGFI la hiperplasia epitelial se encuentra relacionada a una disminución en la tasa de apoptosis asociada al aumento en el número de queratinocitos y también del tejido conectivo, con una disminución de la degradación de matriz extracelular, favoreciendo el acúmulo de colágeno tipo I(13-15). Se ha propuesto que la acumulación de colágeno tipo I es ocasionada por una alteración en el metabolismo del calcio que conduce al desequilibrio entre los niveles de metaloproteinasas y de inhibidores de metaloproteinasas, al igual que un aumento en los niveles de expresión de integrina( ${ }^{(8)}$. En concordancia con lo observado en los casos clínicos y los fármacos consumidos por los pacientes, Gurgel et al., sugieren que medicamentos que provoquen alteraciones en el metabolismo del calcio se asociarían al AGFI, estos generan una disminución del influjo intracelular de calcio llevando a una reducción del ingreso de ácido fólico intracelular, limitando así, la producción de colagenasa activa(16).

Estudios científicos demuestran que el uso de nifedipino, un bloqueador de los canales de calcio empleado para el manejo de enfermedades cardiovasculares, aumenta los niveles de periostina; hecho asociado a elevados niveles de fibrosis ya sea en severidad o en progresión, provocando agrandamiento gingival. De igual manera, estudios de los efectos de la fenitoína in vitro sugieren la presencia de menor producción de colágeno acompañado de un aumento en la densidad del colágeno(1).

Mirabal et al., abordaron tres posibles explicaciones del efecto de los calcioantagonistas con respecto al agrandamiento gingival, las cuales son: diminución de la producción de colagenasas, aumento de la estimulación de la síntesis de colágeno por los fibroblastos gingivales por mediadores específicos y reemplazo de las células inflamatorias por tejido conectivo colágeno. En su reporte de caso, encontraron que después de haber realizado tratamiento profiláctico de higiene oral a paciente con $A G$ inducido por amlodipino y cambiar satisfactoriamente el medicamento, el AG disminuyó considerablemente luego de 4 meses, lo cual se contrasta con el presente caso, debido a que aun utilizando medidas profilácticas de higiene, el AG no cedió y se tuvo que recurrir al procedimiento quirúrgico(17).

El AG se manifiesta como consecuencia de una deficiente higiene oral en el paciente $(4,8)$, así como se pudo observar en los cuatro pacientes, quienes presentaron acúmulo de biopelícula que conlleva a una colonización de bacterias en los tejidos dentarios y gingivales desencadenando un proceso inflamatorio como mecanismo de defensa(1). El tejido gingival ingresa en un ciclo de lesión y remodelación que induce la producción de distintos factores quimiotácticos que influirán en el control del proceso de reparación; se ha demostrado la participación de nifedipino, fenitoína y ciclosporina en la expresión de citoquinas a nivel gingival, sugiriendo un mecanismo de lesión específico en el tejido(18).

La hipertensión arterial es una condición frecuente en la edad adulta consistente en un incremento de la resistencia vascular periférica asociada a una serie de eventos cardiovasculares y complicaciones sistémicas. Los calcioantagonistas son fármacos con efecto hipotensor que ayudan a nivel cardiovascular, a reducir considerablemente esta condición. El amlodipino es un fármaco utilizado para pacientes con hipertensión arterial, es un bloqueador de los canales de calcio de tercera generación que presenta menor efecto con respecto $A G$ a diferencia del nifedipino(5), que se produce un $20 \%$ de los casos; en cambio, el amlodipino sólo presenta una incidencia de agrandamiento gingival en un 3,3(6).

Por el contrario, el agrandamiento gingival inducido por inmunosupresores como las ciclosporinas, altamente utilizadas en casos de trasplantes de órganos, tiene una prevalencia de $40 \%$. Se ha encontrado que el efecto de este fármaco depende de la dosis que ingiere el paciente, a menor dosis, menor efecto(7). La fenitoína, uno de los fármacos más comúnmente utilizado para el tratamiento de pacientes epilépticos debido a su bajo costo y familiaridad, dentro de este grupo de medicamentos es el más 
asociado a AGFI a pesar de no ser el único causante(8).

El abordaje inicial para AGFI consiste en mejorar las estrategias de cepillado e higiene oral con el paciente, acompañado de un tratamiento periodontal no quirúrgico, ya que cuando estos son realizados de forma adecuada y oportuna logran disminuir considerablemente la extensión de la lesión(2,6,8). Si después de haber realizado una correcta fase higiénica periodontal mediante raspado y alisado radicular, profilaxis, técnicas de cepillado y de higiene oral se observa persistencia del AG, se debe recurrir a técnicas quirúrgicas periodontales como gingivectomía y gingivoplastia( $2,3,6,8)$; similar a lo sucedido con los casos clínicos presentados, donde el AG demasiado exacerbado no cedió con la adecuada fase higiénica.

Cabe destacar que la resolución del AG mediante higiene periodontal se empieza a percibir dos o tres meses después de iniciado el

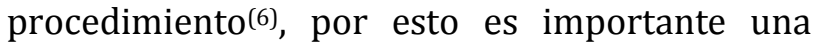
adecuada explicación y comunicación, puesto que existen pacientes que por el aspecto antiestético que este trae consigo, prefieren recurrir al tratamiento quirúrgico como primera instancia, debido a que es una medida rápida y eficaz; sin embargo, se hace necesario explicar las ventajas que se obtienen al aplicar adecuadas técnicas de higiene oral y los resultados de éstos, para así posponer los procedimientos invasivos como último recurso y evitar las complicaciones relacionadas, tales como sangrado, retraso en la cicatrización y riesgo de infección.

La recurrencia del AGFI puede alcanzar incluso un $40 \%$ si se mantiene el uso del medicamento desencadenante, con un tiempo de reaparición entre tres a seis meses después de la intervención quirúrgica, especialmente si el paciente continua con una deficiente higiene oral(2).

Es de importancia resaltar el papel vital que juega el odontólogo con respecto a la presentación del agrandamiento gingival de estos pacientes, puesto que, aunque esta sea una lesión altamente fibrótica también va acompañada de proceso inflamatorio por lo cual un tratamiento periodontal no quirúrgico adecuado y pertinente podría disminuir considerablemente esta lesión(2,6,8). Además, se requiere un trabajo multidisciplinario para evaluar la viabilidad de cambiar o disminuir el fármaco que ingiere el paciente, ya que está confirmado que en cuanto se deje de ingerir estos medicamentos, el agrandamiento gingival también cede e incluso puede desaparecer(1,6).

\section{Conclusión}

El AGFI es una condición frecuentemente observada en clínica, que se presenta secundario a medicamentos de uso rutinario por el personal médico; es de vital importancia realizar un diagnóstico y tratamiento multidisciplinario entre el equipo médico y odontológico para llevar a cabo las intervenciones más adecuadas para cada paciente de forma individual. Cabe resaltar la importancia de los buenos hábitos de higiene oral y la asistencia a citas periódicas con el odontólogo para evitar la acumulación de biopelícula que puede agravar el AGFI. Se debe evaluar la viabilidad de cambiar o disminuir en lo posible la dosis del fármaco asociado para evitar recaídas y complicaciones.

\section{Conflicto de intereses}

No se declaran conflictos de intereses.

\section{Referencias}

1. Straka M, Varga I, Erdelský I, Straka-Trapezanlidis M, Krňoulová J. Drug-induced gingival enlargement. Neuroendocrinology Letters. 2014;35(7):567-76.

2. Livada R, Shiloah J. Calcium channel blocker-induced gingival enlargement. Journal of human hypertension. 2014;28(1):10-4.

3. Eroglu M, Uz O, Isilak Z, Tezcan M, Kilicaslan F, Yiginer O. Nifedipine-induced gingival hyperplasia: an overlooked adverse effect. J Clin Case Rep. 2012;2(225):1-2.

4. Andrew W, Evelyn W, Francis M, Mark J, Mark C. Pattern of Gingival Overgrowth among Patients on Antihypertensive Pharmacotherapy at a Nairobi Hospital in Kenya. Open Journal of Stomatology. 2014;4(4):169-173.

5. Gupta SJ, Madan R, Jhingran R. Drug Induced Gingival Overgrowth with Low-Dose Amlodipine: A Clinical Report. Asian Journal of Oral Health and Allied Sciences. 2013;3(2):81-86.

6. Mathur S, Khatri RK, Mathur R, Srivastava R, Nag B. Drug Induced Gingival Overgrowth: A Rare Case Report. 
Journal of clinical and diagnostic research: JCDR. 2015;9(1):ZD31-ZD33.

7. Wentz LA, Oliveira SC, Moreira CH, Rosing CK. Low prevalence of gingival overgrowth associated to new imunossupressive protocols with cyclosporin. Braz Oral Res. 2012;26(1):64-70.

8. Gurgel BCdV, Morais CRBd, Rocha-Neto PCd, Dantas EM, Pinto LP, Costa AdLL. Phenytoin-Induced Gingival Overgrowth Management with Periodontal Treatment. Brazilian dental journal. 2015;26(1):39-43.

9. Franco V, Perucca E. CYP2C9 polymorphisms and phenytoin metabolism: implications for adverse effects. Expert opinion on drug metabolism \& toxicology. 2015;11(8):1269-79.

10. Calzado de Silva MdlC, Laurencio Rodríguez J, Peña Sisto M. Enfermedades causadas por fármacos en la cavidad bucal. Medisan. 2015;19(11):1386-98.

11. de Asúa DR, Suárez C. Diferencias y similitudes entre los bloqueadores de los receptores del calcio (antagonistas del calcio). Hipertension y riesgo vascular. 2013;30:209.

12. Lores CMQ, Quintero LG, Quintero LG, Quintero RSG, Orduñez YP. Efectividad de Amlodipino en el manejo de hipertensión arterial en población geriátrica. Revista Información Científica. 2015;90(2):209-18.

13. O'Leary TJ, Drake RB, Naylor JE. The plaque control record. J Periodontol. 1972;43(1):38.

14. Pedaballi P, Sundaram R, Ramachandran M. Prevalence of gingival enlargement secondary to calcium channel blockers in patients with cardiovascular diseases. J Indian Soc Periodontol. 2012;16(3):430-435.

15. Trackman P, Kantarci A. Molecular and clinical aspects of drug-induced gingival overgrowth. Journal of dental research. 2015;94(4):540-6.

16. Kim S, Jackson-Boeters L, Darling M, Rieder M, Hamilton D. Nifedipine induces periostin expression in gingival fibroblasts through TGF-beta. Journal of dental research. 2013;92(11):1022-8.

17. Mirabal YÁ, Carbonell LOM. Hiperplasia gingival inducida por amlodipino en paciente con periodontitis crónica. Presentación de un caso. MediCiego. 2016;23(1):36-42.

18. Batista ALA, de Mendonça AKP, de Almeida Freitas R, Alves PM, a Godoy GP, Nonaka CFW, et al. Immunohistochemical Analysis of Cell Proliferation and Bcl-2 Expression in Drug-Induced Gingival Overgrowth. Pesquisa Brasileira em Odontopediatria e Clinica Integrada. 2017;17(1):1-10. 